\title{
Summary of the National Advisory Committee on Immunization's Statement on the re-immunization with polysaccharide 23 -valent pneumococcal vaccine
}

\author{
Quach $C^{1,2}$, on behalf of the National Advisory Committee on Immunization*
}

\author{
${ }^{1} \mathrm{NACl}$ Co-Chair and Pneumococcal Working Group Chair, Montréal, QC \\ ${ }^{2}$ Vaccine Study Centre, McGill University Health Centre, Montréal, QC \\ *Correspondence: naci-ccni@phac-aspc.gc.ca
}

\begin{abstract}
Background: Individuals who are 2 years of age and over and at high risk for invasive pneumococcal disease (IPD) (defined as those with functional or anatomic asplenia or sickle cell disease; hepatic cirrhosis; chronic renal failure or nephrotic syndrome; HIV infection; and immunosuppression related to disease or therapy) are recommended to receive one lifetime booster dose of polysaccharide 23-valent pneumococcal vaccine (Pneu-P23) vaccine, in addition to age- and risk-specific recommendations for the conjugate 13-valent pneumococcal vaccine (Pneu-C-13). Adults aged 65 years and over are also considered at high risk for invasive pneumococcal disease (IPD).

Objective: To determine the optimal time between initial vaccination with Pneu-P-23 and subsequent booster doses to protect against IPD in those at high risk for IPD.

Methods: The National Advisory Committee on Immunization (NACl) conducted a systematic review of the literature on booster doses of pneumococcal vaccine for individuals at high risk for IPD disease. NACl reviewed the evidence considering the target population, safety, immunogenicity, efficacy, effectiveness of the vaccines, vaccine schedules, and other aspects of the overall immunization strategy, and then approved three specific recommendations.
\end{abstract}

Results: For all individuals aged 2 years and over who are at high risk for IPD and who have received a dose of Pneu-P-23, re-vaccination with a second dose of Pneu-P-23 should be provided five years after the initial dose of Pneu-P-23. They should also have previously received age-appropriate doses of 13-valent conjugate pneumococcal vaccine. There is currently insufficient evidence to determine the optimal timing and number of Pneu-P-23 boosters in high-risk adults. One lifetime booster of Pneu-P-23 is currently recommended for individuals at high risk for IPD, five years after the previous dose. Given the increased risk of IPD in adults aged 65 years and older and the rapid decline in antibodies following Pneu-P-23, all individuals should receive one dose of Pneu-P-23 at age 65 years-as long as five years have passed since the previous Pneu-P-23 dose. No additional booster dose is currently recommended for this age group, if they have no medical conditions that put them at high risk for IPD.

Conclusion: The new and complete set of current recommendations for pneumococcal vaccines will be published in the updated "Pneumococcal" chapter in the Canadian Immunization Guide in the near future.

\section{Introduction}

Individuals who are 2 years of age and over and at high risk for invasive pneumococcal disease (IPD) (defined as those with functional or anatomic asplenia or sickle cell disease; hepatic cirrhosis; chronic renal failure or nephrotic syndrome; HIV infection; and immunosuppression related to disease or therapy) should receive one lifetime booster dose of polysaccharide 23-valent pneumococcal (Pneu-P-23) vaccine, in addition to age- and risk-specific recommendations for the conjugate 13-valent pneumococcal vaccine (Pneu-C-13). Adults over 65 years of age are also considered at high risk for IPD, regardless of any medical condition. 
The National Advisory Committee on Immunization (NACl) provides the Public Health Agency of Canada (the Agency) with ongoing and timely medical, scientific, and public health advice relating to immunization. NACl has undertaken a systematic review to determine the optimal time between initial vaccination with Pneu-P-23 vaccine and subsequent booster doses in those at high risk for IPD.

\section{Methods}

$\mathrm{NACl}$ conducted a systematic review of evidence considering the target population, safety, immunogenicity, efficacy, effectiveness of the vaccines, vaccine schedules, as well as other aspects of the overall immunization strategy, and then developed and approved three specific recommendations.

\section{Results}

A total of 10 studies were reviewed for immunogenicity data. Safety of re-vaccination with Pneu-P-23 was assessed in eight of these studies. In all reviewed studies, re-vaccination of those at high risk for IPD five years following initial Pneu-P-23 vaccination demonstrated a boost in immune response and an acceptable safety profile. Individuals who had received one or two doses of Pneu-P-23 before age 65 years demonstrated a good immune response to a repeated dose of Pneu-P-23, when administered at 65 years of age or over. There is little evidence to suggest that hyporesponsiveness occurs with one additional booster of Pneu-P-23. A complete report of the systematic review is available (1).

The following recommendations were developed and approved by $\mathrm{NACl}$.

\section{Updated NACI recommendations on Pneu-P-23 vaccine (2)}

\section{Recommendation \#1}

For all individuals aged 2 years and over who are at high risk for IPD (functional or anatomic asplenia or sickle cell disease; hepatic cirrhosis; chronic renal failure or nephrotic syndrome; HIV infection; and immunosuppression related to disease or therapy) and who have received a dose of Pneu-P-23, re-vaccination with a second dose of Pneu-P-23 should be provided five years after the initial dose of Pneu-P-23. This is a change from the previous recommendation that children aged 10 years or younger at their first dose of Pneu-P-23 should receive the second dose three years later. This change is based on the absence of evidence to support the three-year timing of the booster dose in children and on the universal use of Pneu-C-13 in children that has contributed to the marked decrease in the incidence of IPD. The single re-vaccination at five years after the initial vaccination harmonizes the pediatric and adult schedules for those at high risk for IPD. High-risk individuals should also have received age-appropriate doses of 13-valent conjugate pneumococcal vaccine; 13-valent conjugate pneumococcal vaccine should be administered first, followed eight weeks later by Pneu-P-23.

\section{Recommendation \#2}

There is currently insufficient evidence to determine the optimal timing and number of Pneu-P-23 boosters in high-risk adults (i.e., functional or anatomic asplenia or sickle cell disease; hepatic cirrhosis; chronic renal failure or nephrotic syndrome; HIV infection; and immunosuppression related to disease or therapy). One lifetime booster of Pneu-P-23 is currently recommended for individuals at high risk for IPD, five years after the previous dose.

\section{Recommendation \#3}

Given the increased risk of IPD in adults aged 65 years and older and the rapid decline in antibodies following Pneu-P-23, all individuals should receive one dose of Pneu-P-23 at age 65 years-as long as five years have passed since any previous Pneu-P-23 dose. Studies reviewed for this updated statement have all administered a dose of Pneu-P-23 to individuals aged 65 years and over, regardless of their prior vaccination history. No additional booster dose is currently recommended for those over the age of 65 years who do not have other underlying medical conditions that would put them at high risk for IPD. 


\section{Conclusion}

The new and complete set of current recommendations for pneumococcal vaccines will be published in the updated "Pneumococcal" chapter in the Canadian Immunization Guide in the near future (3). The top surveillance and research priorities are: enhanced surveillance that includes high-risk individuals and can provide incidence of IPD stratified by risk factors and serotypes for individuals in the over 65 years of age group; vaccine effectiveness and coverage studies of Pneu-P-23 in high-risk patients and in those over 65 years of age; and epidemiological studies of non-invasive disease such as community-acquired pneumonia in all age groups or acute otitis media in children caused by S. pneumoniae. To guide recommendations for additional doses (i.e., more than one booster dose), further studies are needed to understand how the immune system responds to additional doses of Pneu-P23.

\section{Acknowledgements}

The author would like to thank the extremely dedicated members of $\mathrm{NACl}$ and the $\mathrm{NACl}$ Pneumococcal Working Group.

\section{Conflict of interest}

None

\section{References}

(1) Caya CA, Boikos C, Desai S, Quach C. Dosing regimen of the 23-valent pneumococcal vaccination: A systematic review. Vaccine. 2015 Mar 10;33(11):1302-12. doi: 10.1016/j.vaccine.2015.01.060. Epub 2015 Feb 3.

(2) National Advisory Committee on Immunization (NACl). Re-Immunization with Polysaccharide 23-Valent Pneumococcal Vaccine (Pneu-P-23): An Advisory Committee Statement (ACS). 2015 April http://publications.gc.ca/site/eng/9.629954/publication.html.

(3) National Advisory Committee on Immunization (NACl). Canadian Immunization Guide. 2015. http://www.phac-aspc.gc.ca/publicat/cig-gci/p04-pneu-eng.php 